\title{
“ANÁLISIS Y EVALUACIÓN DE LA PROMOCIÓN TURÍSTICA EN EL DISTRITO DE CHIGUATA, PROVINCIA DE AREQUIPA 2016".
}

\section{"ANALYSIS AND EVALUATION OF TOURISM PROMOTION IN THE DISTRICT OF CHIGUATA, PROVINCE OF AREQUIPA 2016".}

\author{
Mg. CAPPILLO SALAZAR, IVÁN / SHAONI JESÚS COSTA CARBAJAL
}

\section{RESUMEN}

Objetivo: La presente investigación tiene como objetivo evaluar la promoción turística en el distrito de Chiguata, provincia de Arequipa 2016. Materiales y métodos: La investigación es aplicada de acuerdo al nivel de investigación descriptiva con un diseño Descriptivo - Simple (no experimental), porque analizaremos la promoción del Distrito de Chiguata, mediante su página web con el fin de generar estrategias para que se promocione este destino eficazmente a través de este medio. El tipo de investigación es, Sustantiva Descriptiva, el corte del tipo de investigación: es transversal, porque solo se dará en un momento dado. Se trabajó con una muestra de 70 personas en la cual se realizó mediante el criterio de selección, con ciertas características correspondientes, para desarrollar unas encuestas acerca de la promoción de los atractivos naturales y culturales. La técnica a emplearse en la presente investigación será la encuesta por medio de cuestionarios. Resultados: De los 70 turistas encuestados que equivalen a un $100 \%$, se puede observar, que la mayoría de personas con un $31,4 \%$, no tienen mucho conocimiento en las redes sociales, es por eso que, no comentan acerca de sus viajes o experiencias, ya que, al no tener ese conocimiento, no conocerán las buenas funciones que se puede obtener mediante el internet. Otros dicen que la misma página del lugar, no encuentra actualizada, con un 32,9\%, siendo, muy escaza la información que se puede brindar acerca de sus atractivos turísticos, programas o campañas. Conclusiones: Se concluye que el 31,4 \% de los turistas nacionales, no tienen mucho conocimiento en las redes sociales, es por eso que, no comentan acerca de sus viajes o experiencias, ya que al no tener ese conocimiento, no conocerán las buenas funciones que se puede obtener mediante el internet. Otros dicen que la misma página del lugar, no se encuentra actualizada, es por eso, que se obtiene un 32,9\%, siendo, muy escaza la información que se puede brindar acerca de sus atractivos turísticos, programas o campañas, ya que existen dificultades, en la promoción, por un mal manejo de las entidades de turismo, tanto por la oficina de turismo y la municipalidad del lugar. Según los autores, afirman 
que, para promocionar un destino será necesaria la difusión, mediante página web, marketing, con el objetivo de atraer a los turistas, y lograr satisfacer sus exigencias requeridas. Según: Ejarque (2005) define la promoción de un destino como "comunicar a los potenciales consumidores-turistas que la oferta propuesta es capaz de satisfacer sus exigencias y sus demandas; en definitiva, es tratar de convencer al turista de que merece la pena ir a un destino, visitarlo".

Palabras claves: Promoción, marketing, folletos, difusión, internet.

\section{ABSTRACT}

Objective: The objective of this research is to evaluate tourism promotion in the district of Chiguata, province of Arequipa 2016. Materials and Methods: The research is applied according to the level of descriptive research with a Descriptive - Simple (non - experimental) design, because we will analyze the promotion of the District of Chiguata, through its web page in order to generate strategies to promote this destination effectively through Of this medium. The type of research is, Descriptive Noun, the cut of the type of investigation: it is transversal, because it will only occur at a given moment. We worked with a sample of 70 people in which it was done using the selection criteria, with certain characteristics, to develop surveys about the promotion of natural and cultural attractions. The technique to be used in the present investigation will be the questionnaire survey. Results: Of the 70 tourists surveyed that are equivalent to $100 \%$, it can be observed that most people with $31.4 \%$ do not have much knowledge in social networks, that is why, they do not comment on their trips or experiences, Since not having that knowledge, will not know the good functions that can be obtained through the internet. Others say that the same page of the site, does not find updated, with $32.9 \%$, being very scarce the information that can be provided about its tourist attractions, programs or campaigns. Conclusions: It is concluded that $31.4 \%$ of national tourists do not have much knowledge in social networks, that is why, they do not comment on their travels or experiences, because they do not have that knowledge, they will not know the good functions that Can be obtained through the internet. Others say that the same page of the site, is not updated, that is why, you get a $32.9 \%$, being very scarce information that can be provided about its attractions, programs or campaigns, as it exists Difficulties, in the promotion, by a mismanagement of the tourism entities, both by the tourist office and the local municipality. According to the authors, they affirm that, in order to promote a destination, it will be necessary to disseminate, through 
a website, marketing, with the aim of attracting tourists, and achieving their required requirements. According to: Ejarque (2005) defines the promotion of a destination as "to communicate to potential consumers-tourists that the proposed offer is capable of satisfying their demands and demands; In short, is to try to convince the tourist that it is worth going to a destination, to visit it. "

Keywords: Promotion, marketing, brochures, broadcast, internet.

\section{Mg. Cappillo Salazar, Iván}

Docente de la Facultad de Ciencias Empresariales y Educación - Universidad Alas Peruanas. Shaoni J. Costa Carbajal

Estudiante de la EAP de Turismo, Hotelería y Gastronomía - Universidad Alas Peruanas.

\section{INTRODUCCIÓN}

En la actualidad, podemos observar que, en este lugar, no hay promoción ni difusión por las entidades (municipio), debido a que los turistas desconocen este lugar, por la falta de información (páginas web), no hay un debido mantenimiento en esas páginas, falta de apoyo por las entidades de turismo, y falta un inventario turístico actualizado de los atractivos, tanto naturales como culturales, etc.

La presente investigación se elaboró con la finalidad de atraer más turistas al lugar, dando a conocer sus atractivos turísticos tanto natural como cultural, mediante los instrumentos, como volantes, afiches, para promocionar este lugar. Y de esta esta manera el turista pueda informarse mejor, que no lo solo al visitar la ciudad de Arequipa, también pueda conocer sus distritos como Chiguata. Además, al informarse y conocer mejor el lugar, este turista puede comentarles a sus amigos, familiares, y así, se daría a conocer más el Distrito de Chiguata.

Morgan et Pritchard (1999). "Indican que también se debe difundir determinada información, desarrollar ciertas actitudes y promover acciones que beneficien a la comunidad receptora. En estos últimos años, la promoción turística ha comenzado una nueva fase basada principalmente en la web, fruto de la evolución natural promovida por el marketing y por las nuevas tecnologías de comunicación”.

Este lugar no es muy conocido por los turistas, y es por eso que se realiza, esta investigación. Pero nos ponemos a pensar ¿Por qué el distrito de Chiguata, no es muy 
conocido, si fue creado antes que la Ciudad de Arequipa?

La interrogante es porque la mayoría de agencias de viajes, no crean paquetes turísticos de distritos, si poseen atractivos que pueden darse a conocer, y aumentar el flujo de turistas al lugar.

Como consecuencia de la investigación, se deduce la poca publicidad y promoción que existe, ya que, no se llega a conocer en su totalidad como debería de ser.

Acerenza (1990) la promoción turística debe lograr que los consumidores conozcan los atractivos y la infraestructura existente en la localidad que diferencie el destino de su competencia y que inspire confianza y credibilidad, además de influir en la elección del destino y la decisión de compra.

En Guayaquil, Según la bachiller Robalino M., (2012), llego a la conclusión, que mediante la realización de esta promoción Turística al balneario de Banchal se incrementará el estatus económico de los pobladores, en los cuales se dará un buen en el trato al turista y les ayudará a mejorar sus servicios, para lo cual el Municipio debería encargarse de la capacitación. Además la creciente demanda ayudará a producir un efecto multiplicador en la población. Y así, se podrá captar la atención de operadores turísticos grandes que incluso podrían iniciar el flujo de turistas extranjeros al cantón.
La presente investigación tiene una justificación social porque se posicionar el Distrito de Chiguata, como una alternativa de turismo cultural y de naturaleza, favoreciendo el intercambio cultural entre pobladores y turistas, manifestando las culturas, costumbres, idiomas, etc., y de esta manera podemos incrementar la promoción mediante los turistas ya que al relacionarse con los pobladores, se conocerá más el lugar y así ese turista, atraerá a más personas. De esta manera, será más conocido el lugar y se incrementará el turismo.

Por último, esta investigación pretende demostrar, que tanta promoción existe, y de qué manera se puede aumentar el flujo de turistas. Ya que el objetivo general evaluar la promoción turística en el distrito de Chiguata, provincia de Arequipa 2016.

\section{MATERIALES Y MÉTODOS}

Se trabajó con una muestra de 70 personas en la cual se realizó mediante el criterio de selección, con ciertas características correspondientes, para desarrollar unas encuestas acerca de la promoción de los atractivos naturales y culturales. El diseño utilizado para la investigación es diseño Descriptivo - Simple (no experimental), porque analizaremos la promoción del Distrito de Chiguata, mediante su página web con el fin de generar estrategias para 
que se promocione este destino eficazmente a través de este medio.

Según Palella et Martins (2004), expresan que el diseño de la investigación "se refiere a la estrategia que adopta el investigador para responder al problema, dificultad o inconveniente planteado en el estudio".

El tipo de investigación es, Sustantiva Descriptiva, el corte del tipo de investigación: es transversal, porque solo se dará en un momento dado.

Según Fiallo Rodríguez J.P. y otros (2008).La investigación sustantiva, "Es aquella que trata de responder a los problemas teóricos o sustantivos específicos, en tal sentido, está orientada, a describir, explicar, predecir o retrodecir la realidad, con lo cual se va en búsqueda de principios y leyes generales que permita organizar una teoría científica. En este sentido, podemos afirmar que la investigación sustantiva al perseguir la verdad nos encamina hacia la investigación básica o pura”.

Y el nivel de Investigación Descriptiva, porque de acuerdo al problema planteado, referido a la deficiente promoción del lugar, será necesario realizar una investigación proyectiva para proporcionar distintas soluciones mediante el mantenimiento de su página social (Facebook).
Según Sánchez Carlessi H. y Reyes Meza C. (2006) manifiestan que siguiendo a Selltiz, Jahoda y otros (1965), podemos identificar tres niveles o esquemas básicos de investigación (según la profundidad y objetivo) que pueden relacionarse a los tipos de investigación anteriormente descritos. Estos son: Investigación descriptiva, "Tiene como objetivo la descripción de los fenómenos a investigar, tal como es y cómo se manifiesta en el momento (presente) de realizarse el estudio y utiliza la observación como método descriptivo, buscando especificar las propiedades importantes para medir evaluar aspectos, dimensiones o component es

La técnica utilizada para esta investigación es la encuesta, y el instrumento a emplear es el cuestionario para evaluar la promoción a través de los turistas. Los datos fueron recopilados y analizados en el paquete estadístico o software el SPSS versión 24.

\section{RESULTADOS}

De los 70 turistas encuestados que equivalen a un $100 \%$, se puede observar, que la mayoría de personas con un $31,4 \%$, no tienen mucho conocimiento en las redes sociales, es por eso que, no comentan acerca de sus viajes o experiencias, ya que al no tener ese conocimiento, no conocerán las 
buenas funciones que se puede obtener mediante el internet. Otros dicen que la misma página del lugar, no encuentra actualizada, con un $32,9 \%$, siendo, muy escaza la información que se puede brindar acerca de sus atractivos turísticos, programas o campañas. (Fig. 1).

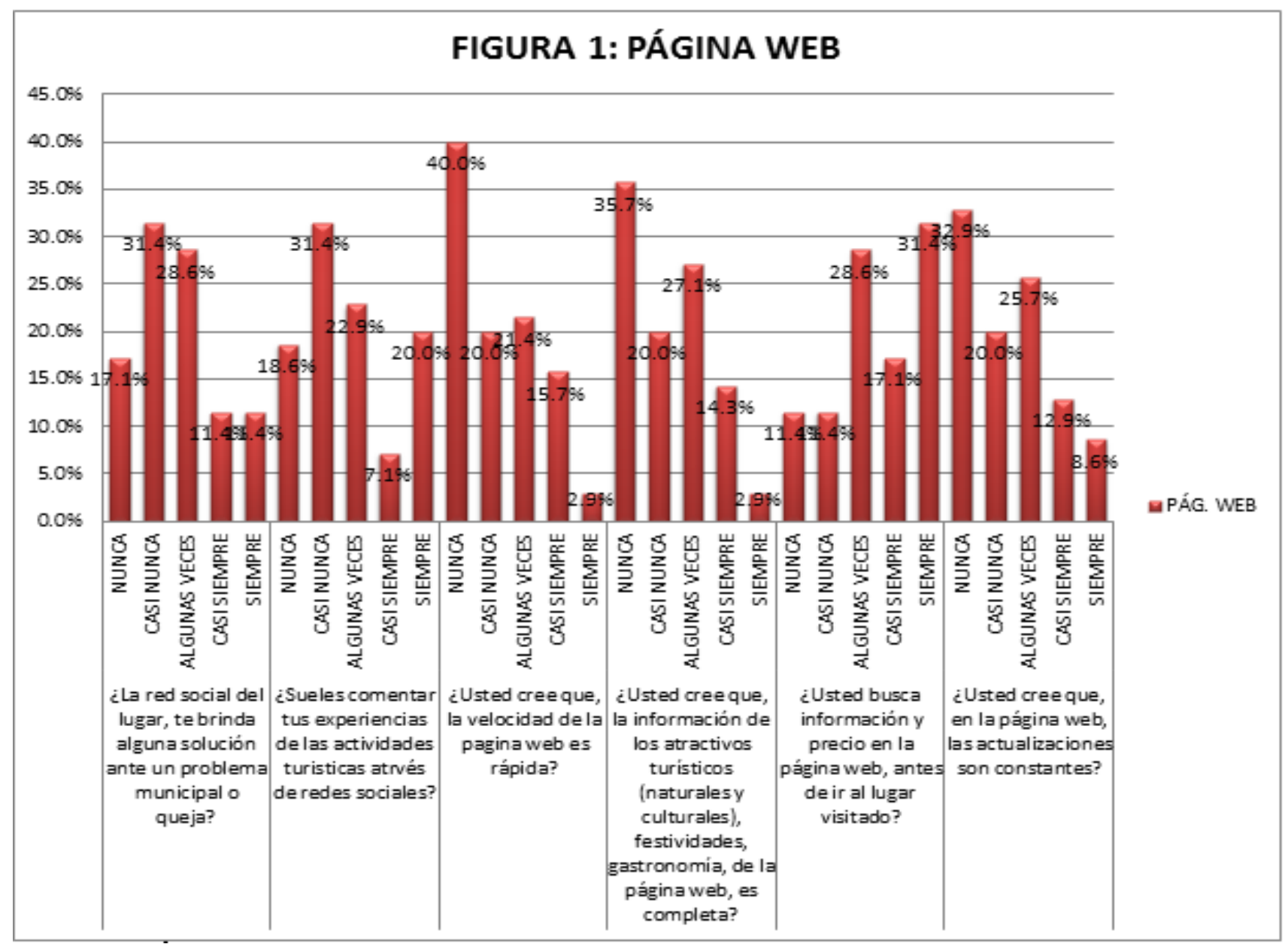

\section{Fuente: Elaboración Propia}

\section{DISCUSIÓN}

Como se mencionó anteriormente, se desconoce mucho sobre el tema, porque no existe una adecuada información, acerca de los atractivos naturales y culturales, que posee el distrito de Chiguata.
En el grafico $\mathrm{n}^{\circ}$ 1: De los 70 turistas encuestados que equivalen a un $100 \%$, se puede observar, que la mayoría de personas con un $31,4 \%$, no tienen mucho conocimiento en las redes sociales, es por eso que, no comentan acerca de sus viajes o experiencias, ya que al no tener ese 
conocimiento, no conocerán las buenas funciones que se puede obtener mediante el internet. Otros dicen que la misma página del lugar, no encuentra actualizada, con un $32,9 \%$, siendo, muy escaza la información que se puede brindar acerca de sus atractivos turísticos, programas o campañas.

Estas conclusiones son similares a los de la Bachiller García Camayo, z. p. "Promoción turística de la región Junín a través del gobierno regional y gobiernos locales, para optar el título profesional de licenciado en turismo, hotelería y gastronomía, universidad alas peruanas - facultad de ciencias empresariales, 2013", cuyas conclusiones fueron: La existencia de dificultades en la promoción turística de la región Junín a través del gobierno regional y local, mediante el cual, existen empirismos aplicativos, al no aplicar adecuadamente los conceptos, principios o estrategias que permitan un mejor manejo de la actividad turística por parte del sector público, o por presentar carencias en los productos turísticos y/o en la prestación de servicios turísticos de calidad.

Acerenza. En su libro "Promoción turística" (s.f.), “dice que la promoción turística comprende las actividades emprendidas con el objeto de presentar un mensaje o una idea del producto a un grupo de personas atreves de la difusión".
Según García. J, (2012). "La promoción turística consiste en dar a conocer un destino que posea todo lo referido al interés del turismo fomentando el desarrollo en el turismo rural, ecoturismo brindándole al visitante una nueva alternativa el cual tenga contacto con la naturaleza, la aventura, el descanso y el entretenimiento".

Según Ejarque (2005), define la promoción de un destino como “comunicar a los potenciales consumidores-turistas que la oferta propuesta es capaz de satisfacer sus exigencias y sus demandas; en definitiva, es tratar de convencer al turista de que merece la pena ir a un destino, visitarlo".

El resultado del estudio concuerda con García y Ejarque ya que hoy en día, para dar a conocer algún destino, será necesario promocionarlo, crear paquetes turísticos, que incluyan visitas a los distritos, con precios accesibles para la totalidad de turistas.

\section{CONCLUSIONES}

Se concluye que el $31,4 \%$ de los turistas nacionales, no tienen mucho conocimiento en las redes sociales, es por eso que, no comentan acerca de sus viajes o experiencias, ya que al no tener ese conocimiento, no conocerán las buenas funciones que se puede obtener mediante el 
internet. Otros dicen que la misma página del lugar, no se encuentra actualizada, es por eso, que se obtiene un $32,9 \%$, siendo, muy escaza la información que se puede brindar acerca de sus atractivos turísticos, programas o campañas, ya que existe dificultades, en la promoción, por un mal manejo de las entidades de turismo, tanto por la oficina de turismo y la municipalidad del lugar.

Según los autores, afirman que, para promocionar un destino será necesaria la difusión, mediante página web, marketing, con el objetivo de atraer a los turistas, y lograr satisfacer sus exigencias requeridas. Según: García. J, (2012). La promoción turística consiste en dar a conocer un destino que posea todo lo referido al interés del turismo fomentando el desarrollo en el turismo rural, ecoturismo brindándole al visitante una nueva alternativa el cual tenga contacto con la naturaleza, la aventura, el descanso y el entretenimiento. $\mathrm{Y}$ según Ejarque (2005) define la promoción de un destino como "comunicar a los potenciales consumidores-turistas que la oferta propuesta es capaz de satisfacer sus exigencias y sus demandas; en definitiva, es tratar de convencer al turista de que merece la pena ir a un destino, visitarlo".

\section{REFERENCIAS BIBLIOGRÁFICAS}

1. Morgan et Pritchard (1999, p.57). Promoción Turística. Recuperado de: http://www.estudiosenturismo.com.ar/sea rch/PDF/v17n1y2a8.pdf

2. Acerenza (1990, p.691). La promoción turística. Recuperado de: http://www.scielo.org.ar/pdf/eypt/v19n5/ v19n5a06.pdf

3. Robalino, M. G. A., (2012, p.63). Tesis "Promoción Turística del Balneario de Banchal Parroquia Cascol Cantón Paján Provincia De Manabí”. Recuperado de: http://repositorio.ug.edu.ec/bitstream/red ug/1401/1/TESIS\%20PROMOCION\%20 TURISTICA\%20DEL\%20BALNEARIO \%20DE\%20BANCHAL\%20$\%$ 20GISELLA.pdf

4. Palella y Martins (2004: Párr.18). Diseño de la Investigación. Recuperado de:

http://www.eumed.net/tesisdoctorales/2012/zll/metodologiainvestigacion.html

5. Fiallo Rodríguez J.P. y otros (2008, Párr.8). La Investigación sustantiva del Tipo de Investigación. Recuperado de: https://es.scribd.com/doc/97318021/Tipo s-y-Niveles-de-Investigacion-Cientifica

6. Sánchez Carlessi H. Et Reyes Meza C. (2006, Párr.15). Los tipos de investigación. Recuperado de: https://es.scribd.com/doc/97318021/Tipo s-y-Niveles-de-Investigacion-Cientifica

7. García Camayo, Z. P. (2013). Tesis "Promoción Turística de la Región Junín a través del Gobierno Regional Y Gobiernos Locales". 
Biblioteca de la Universidad Alas Peruanas

8. Acerenza (s.f., P.22). En su libro “Promoción turística". Recuperado de:

http://repo.uta.edu.ec/bitstream/12345678 9/9315/1/FCHE-HTP-194.pdf

9. García. J, (2012, P.10). Promoción Turística. Recuperado de:

http://repositorio.ug.edu.ec/bitstream/red ug/9771/1/PROPUESTA\%20DE\%20UN \%20PLAN\%20DE\%20MARKETING\%2 OPARA\%20UN\%20COMPLEJO\%20TU R\%C3\%8DSTICO.pdf

10. Ejarque (2005, P.739). Promoción Turística. Recuperado de: http://www.redalyc.org/pdf/1807/180739 769017.pdf 